\title{
Surgical treatment of the primary tumour improves the overall survival in patients with metastatic breast cancer: A systematic review and meta-analysis
}

\author{
HANNAH HEADON, UMAR WAZIR, ABDUL KASEM and KEFAH MOKBEL
}

The London Breast Institute, Princess Grace Hospital, London W1U 5NY, UK

Received August 17, 2015; Accepted January 20, 2016

DOI: $10.3892 / \mathrm{mco} .2016 .778$

\begin{abstract}
Traditionally, stage IV metastatic breast cancer has been treated with systemic therapy and/or radiotherapy in order to decrease cancer-associated symptoms, maintain quality of life and control disease burden. Previous research suggests that surgical treatment of the primary tumour may prolong survival, as well achieve local control of disease. Using the PubMed and Ovid SP databases, a literature review and meta-analysis was performed in order to assess whether surgical resection of the primary tumour in metastatic breast cancer prolongs survival. In this meta-analysis, a pooled hazard ratio of 0.63 (95\% confidence interval, $0.58-0.7$; $\mathrm{P}<0.0001$ ) was revealed, equating to a $37 \%$ reduction in risk of mortality in patients that underwent surgical resection of the primary tumour. Therefore, it was concluded that surgery of the primary tumour in stage IV breast cancer appears to offer a survival benefit in metastatic patients.
\end{abstract}

\section{Introduction}

A small percentage of patients presenting with breast cancer are found to have metastatic disease at the point of presentation. Breast cancer, with distant metastases beyond the regional lymph basin, remains a therapeutic challenge. The mainstays of therapy in such advanced disease are systemic therapies, including chemotherapy or endocrine therapy, or palliative loco-regional strategies, including targeted radiotherapy or surgery to metastases (1). Current recommendations for treatment of such advanced stage IV disease include no curative resection, as evidence of a survival benefit for primary resection in such cases is lacking (2).

However, recent previous studies suggest that primary tumour resection may be an independent factor in improving

Correspondence to: Professor Kefah Mokbel, The London Breast Institute, Princess Grace Hospital, 45 Nottingham Place, London W1U 5NY, UK

E-mail: kefahmokbel@hotmail.com

Key words: breast cancer, metastasis, systematic review, meta-analysis, primary resection, mastectomy survival and in addition, control of local symptoms $(3,4)$. It has been suggested that resection of the primary tumour in stage IV disease aids survival by reducing the tumour burden, specifically the number of circulating tumour cells (5). Additionally, recent evidence have postulated a model in which metastases can 'self-seed' and circulate back to the primary tumour, accelerating growth and angiogenesis through cytokine action (6). Furthermore, the role of the primary tumour in advanced disease is a central theme of currently debated disease models centred around the postulated role of cancer stem cells (7).

However, a consensus regarding a curative role for primary resection in stage IV disease remains to be determined, as the relevant evidence is far from unanimous. It is suggested that the beneficial effect observed in other previous studies may be the result of a selection bias.

In order to better examine this issue, the present study performed a systematic review of the literature and a meta-analysis in order to calculate the survival benefit of primary resections in stage IV breast cancer.

\section{Materials and methods}

Data sources and searches. A comprehensive search of the PubMed (http://www.ncbi.nlm.nih.gov/pubmed) and Ovid SP (http://ovidsp.uk.ovid.com/sp-3.18.0b/ovidweb.cgi) databases was performed to identify relevant published literature prior to February $25^{\text {th }} 2015$. The search keywords used were as follows: 'Stage IV breast cancer' and 'surgical excision' or 'surgery' or 'local treatment'.

The authors as per pre-specified inclusion and exclusion criteria assessed the articles identified.

Inclusion and exclusion criteria. Prospective clinical trials and retrospective case series regarding female adult patients with reported outcomes as a function of surgical resection of primary breast cancer in the presence of histologically confirmed distant metastases were included. Conservative and extended resections were included, with no stipulations regarding systemic therapies and the use of radiation or surgery in the regional lymph basin.

The exclusion criteria were as follows: i) Studies reporting no hazard ratios (HRs) for overall survival of adult female patients, according to multivariate analysis; ii) studies that 


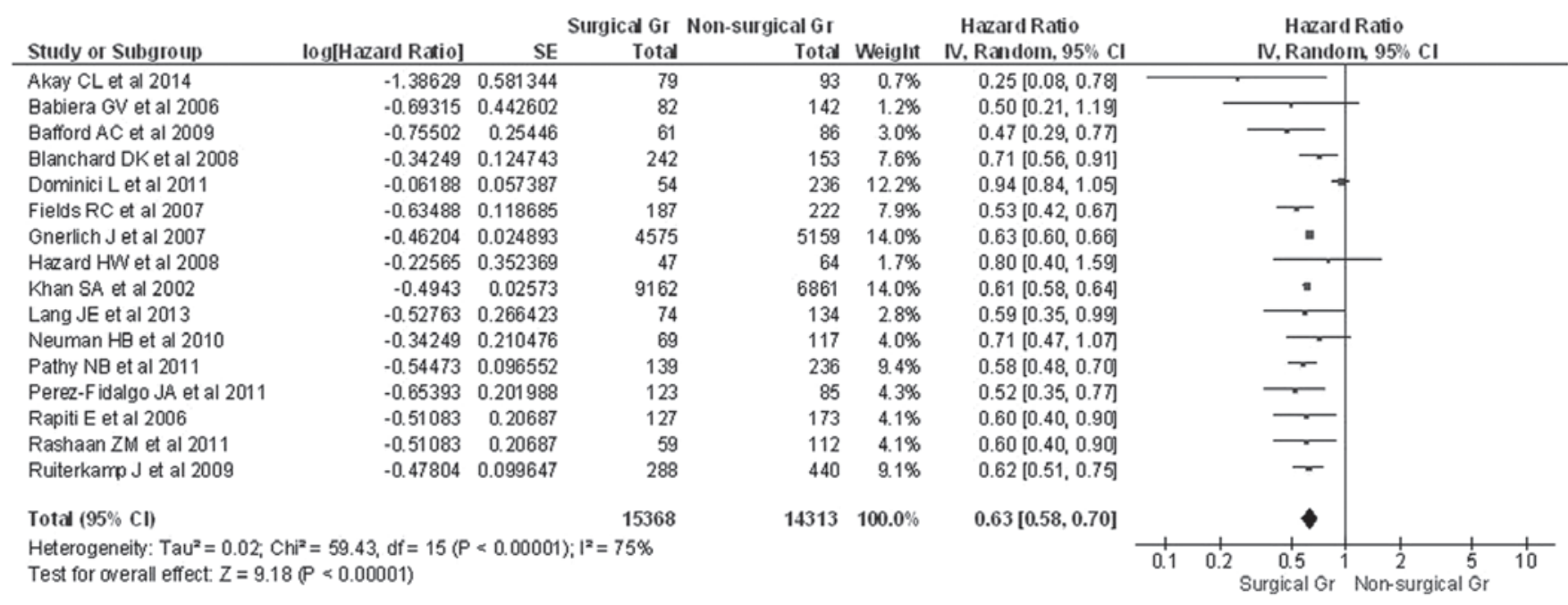

Figure 1. Forest plot of hazard ratios and pooled analysis for overall mortality for surgery, vs. no surgery in patients with stage IV breast cancer.

failed to report $95 \%$ confidence intervals (CIs) for the HRs; iii) unavailability of full text for data extraction; iv) reviews, case reports, letters or commentaries.

Data extraction and management. Data was extracted by the authors independently using characteristics of included studies, the baseline characteristics of included patients and the aforementioned outcomes. The recorded data included author, publication date, study design, participants, interventions, median age, oestrogen receptor (ER) status, human epidermal growth factor receptor 2 status and metastatic sites.

Measures of treatment effect and statistical analysis. HRs and CIs for overall survival as a function of surgery of primary breast cancer with or without other treatment modalities were retrieved for each study. An $\mathrm{HR}<1$ meant a reduced risk of mortality for surgically treated patients compared with those who did not undergo primary tumour resection.

A meta-analysis of HRs was performed with both fixed effect and random effect models considered. Statistical heterogeneity among the included studies was assessed using Cochran's Q test, and a $\chi^{2}$ test and $\mathrm{I}^{2}$ statistic was used to quantify the inconsistency: A value of $0-100 \%$ indicated increasing heterogeneity. The assumption of homogeneity was considered invalid for $\mathrm{P}<0.1$. Summary estimates were reported from the random-effects models.

Potential publication biases were evaluated with Begg's funnel plots for overall survival and subsequently with both Begg's and Egger's tests. Duval and Tweedie's trim and fill method was also performed.

Results of the meta-analyses were reported as a classical forest plot. Statistical analyses were performed using Review Manager 5.1 and Comprehensive Meta-Analysis version 3.0.

\section{Results}

Literature search results and the characteristics of the included studies. A total of 1,628 studies were retrieved, of which 19 initially met the inclusion criteria. Of these studies, two were excluded at the end of the selection phase due to a lack of HRs for overall survival (OS) in the multivariate analysis. One additional study was excluded as it failed to report a 95\% CI for HR, thus precluding calculation of the standard error for meta-analysis. Therefore, 16 studies met the full inclusion criteria for this meta-analysis (Table I) (2-4,8-20). All previous studies were retrospective case studies.

Meta-analysis for $O S$. The present study first tested the overall null hypothesis, which stated that all treatment effects equalled zero. This is equivalent to testing whether all HRs in all studies are equal to 1 , indicating no effect from surgery. Both non-directional and directional tests rejected the null hypothesis.

The HRs for OS and standard errors for the estimated HRs were reported or extrapolated for all included studies. Significant heterogeneity was observed by calculating the $\chi^{2}$ test for heterogeneity $(\mathrm{P}<0.0001)$ and the $\mathrm{I}^{2}$ test demonstrated an index of $75 \%$, indicating considerable inconsistency between the selected studies. Therefore, the present study assumed a random effects model that takes into account variability within and between studies. The pooled HR for OS was 0.63 with a $95 \% \mathrm{CI}$ of $0.58-0.70$ (Table I), confirming the suggestion that surgery is beneficial in terms of reducing the risk of mortality by $37 \%$. These results all illustrated in the forest plot in Fig. 1.

The funnel plot for risk of bias in OS (Fig. 2) revealed that all studies, with the exception of Dominici et al (10), fall within the $95 \% \mathrm{CI}$, and they are relatively symmetrically distributed. Therefore, it would be reasonable to surmise that there are significant systematic differences between the individual studies.

Evidence of publication bias was not revealed in the present analysis, despite the use of multiple tests for this purpose (Egger's test, $\mathrm{P}=0.40785$; Begg test, $\mathrm{P}=0.50$ Mazumdar's rank correlation test, $\mathrm{P}=0.50)$.

According to Duval and Tweedie's 'trim and fill' method under the random-effect model (point estimate $=0.64674 ; 95 \% \mathrm{CI}=0.58774-0.71167)$, the imputed point estimate for OS remained unchanged. 


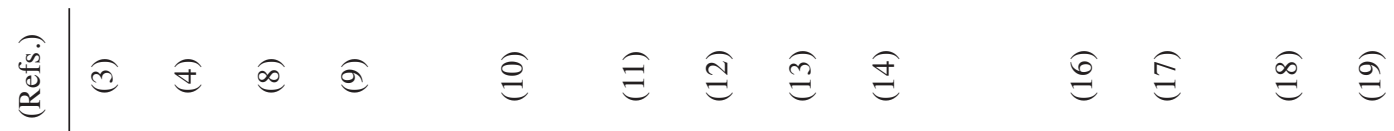

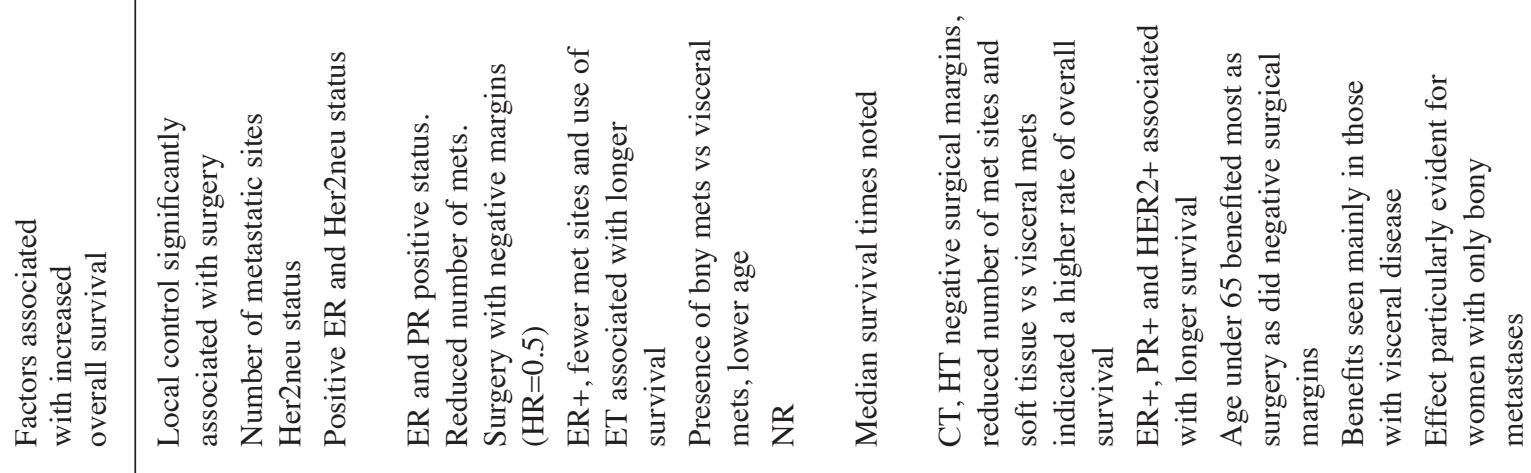

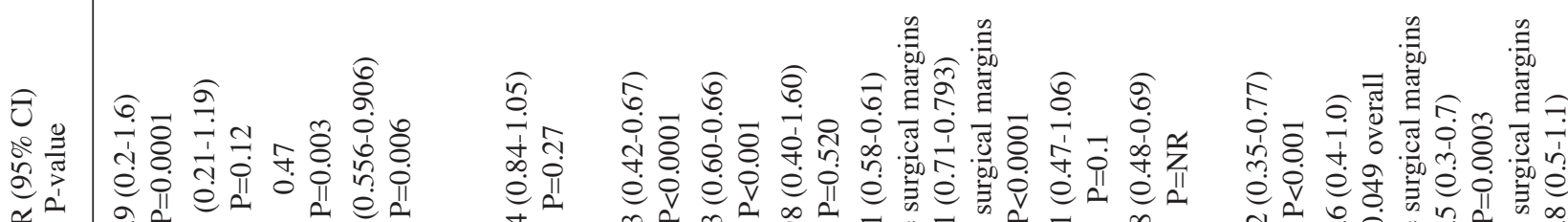

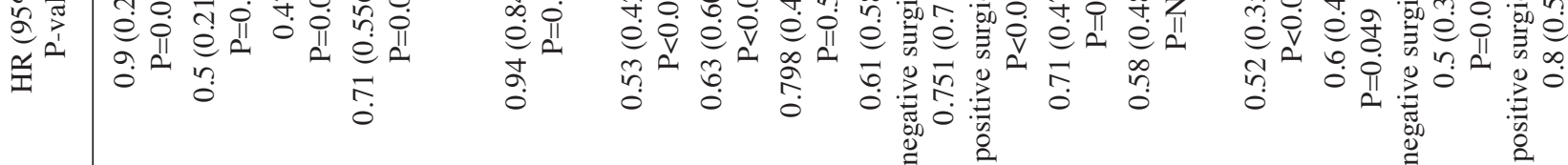

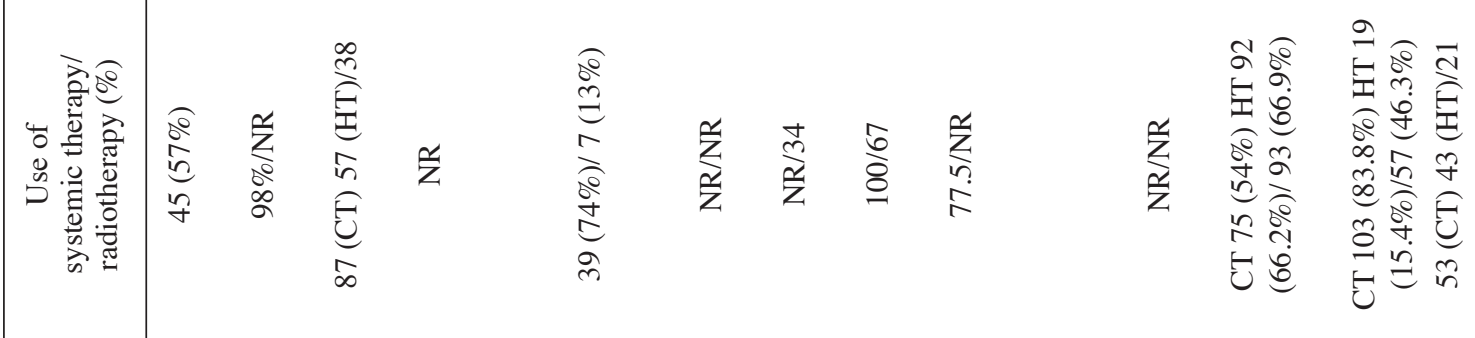

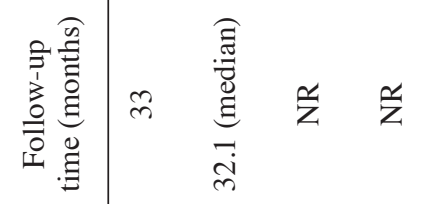

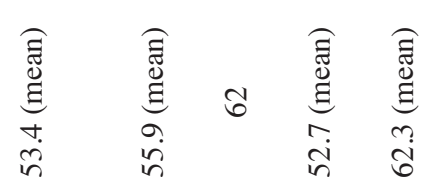

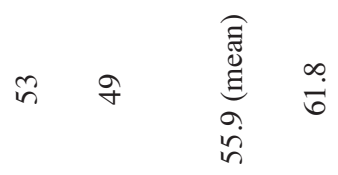

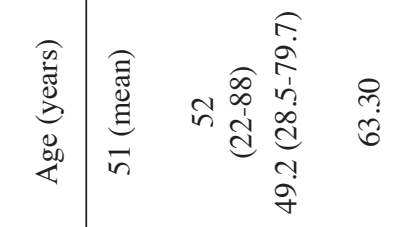

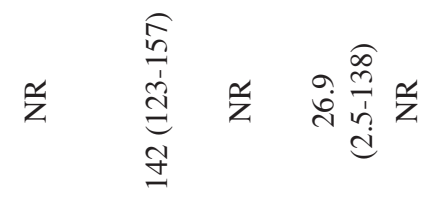

ก $\frac{1}{z} \stackrel{\infty}{\infty} \frac{a}{z}$

$\dot{\dot{z}} \dot{\mathrm{O}} \overrightarrow{\mathrm{g}}$

(5)

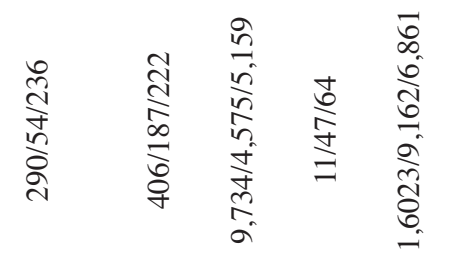

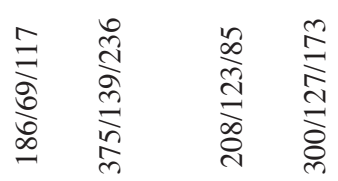

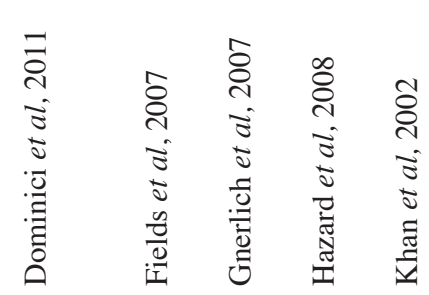

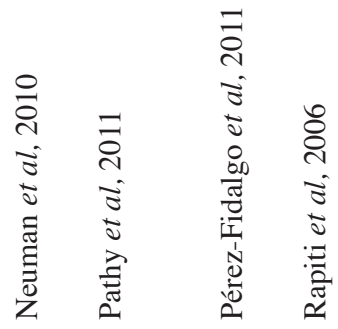




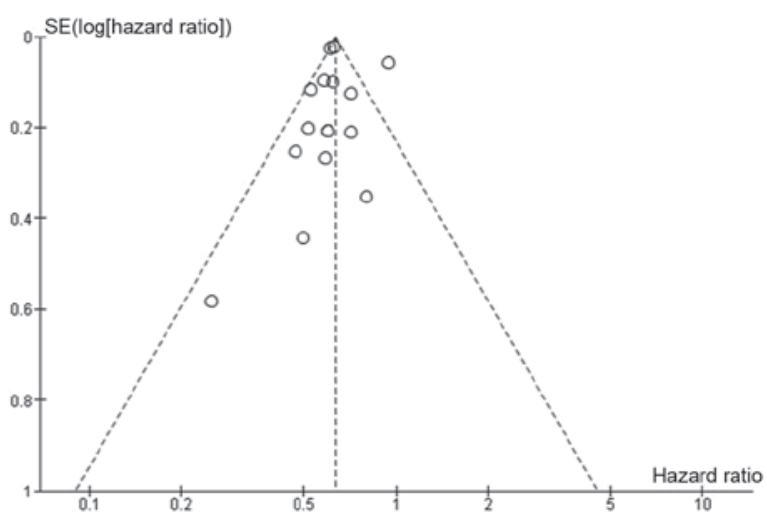

Figure 2. Funnel plot for overall survival meta-analysis. All previous studies, with the exception of that by Dominici et al (10), were within the 95\% confidence intervals and were relatively symmetrically placed.

\section{Discussion}

The present meta-analysis confirmed the hypothesis that resection of the primary tumour in a patient with concomitant metastatic disease is beneficial in terms of survival, with a $37 \%$ reduction in mortality. These results reiterated the benefits of surgical resection of the primary tumour in metastatic disease, not just for advanced breast cancer, but also potentially for other advanced cancer types.

A number of hypotheses can be postulated regarding the mechanisms underlying the beneficial effects on prognosis of primary resection in metastatic breast cancer. Aside from the self-evident role of reducing the overall tumour burden, removal of the primary tumour has been shown to reduce the number of circulating tumour cells, which has been be associated with improved disease outcomes (5).

Furthermore, recent previous studies describe a disease model termed 'tumour self-seeding', in which the primary tumour may release cells into the circulation to seed metastases, which in turn seed the primary tumour, leading to more virulent disease (6).

Additionally, some of the suggested effects of primary resections may be explicable under the currently topical cancer stem cell model, in which metastatic disease is postulated as a systemic disorder orchestrated by a more finite number of stem cells within the primary tumour, which recruit further cells by maintaining an oncogenic microenvironment $(7,21)$. Furthermore, primary resection of tumours may assist in restoring an immunocompetent status by reactivating autoimmunity, therefore increasing the efficacy of any concomitant medication despite the presence of metastatic disease (22). Whist this model remains highly contentious, the findings of the present study are highly suggestive of an active role of the primary tumour in metastatic disease, which suggests a role for surgery in this context in addition to palliation and local symptom control.

A number of the previous studies included here highlighted additional positive prognostic factors in terms of OS in the course of univariate analysis. The most common were: A reduced number of metastatic sites ('oligometastatic state'); positive ER status; a younger age; a smaller primary tumour $(2,4,8-11,16,17,20,23)$. Additionally, Pathy et al (19) 
observed that patients with positive margins received no benefit from resection in terms of OS (17). Rapiti et al (19) reported similar findings in the course of their study (19). This may have important implications regarding the surgical treatment that would be beneficial in this patient group. Furthermore, it raises the question whether a mastectomy would be a more appropriate intervention compared with tumour resection. Finally, the timing of the surgery in relation to adjuvant and neo-adjuvant therapies remains an area of uncertainty. These questions fall out of the remit of this meta-analysis and are worthy issues for exploration in course of future prospective studies.

A major limitation of this meta-analysis is that the paucity of prospective data in the available literature. Furthermore, despite adjusting for heterogeneity through use of random-effect modeling, a high level of inconsistency remains. Another limitation that must be acknowledged was the lack specificity regarding the non-surgical treatment administered (i.e., whether the patients underwent chemotherapy, radiotherapy, endocrine therapy or a combination). Finally, patient populations that underwent surgery were predominantly younger, therefore precluding comparison with other patient groups.

Prospective data would be required to confirm or refute the present findings. One ongoing trial may answer some of these questions. It is randomised cohort trial comparing immediate resection of the primary tumour, followed by systemic therapy and systemic therapy, followed by delayed surgical resection (24). It is prudent to revise this meta-analysis when the results of this and other similar trials become available.

Whilst acknowledging the limitations of this study, the present findings are highly suggestive of a significant benefit for resection of the primary tumour in advanced metastatic breast cancer, and would support a discussion regarding the inclusion of primary resection in the treatment options offered to the patient.

\section{Acknowledgements}

The present study was funded by grants from the Breast Cancer Hope Foundation (London, UK).

\section{References}

1. Petrelli F and Barni S: Surgery of primary tumors in stage IV breast cancer: An updated meta-analysis of published studies with meta-regression. Med Oncol 29: 3282-3290, 2012.

2. Ruiterkamp J, Voogd AC, Bosscha K, Tjan-Heijnen VC and Ernst MF: Impact of breast surgery on survival in patients with distant metastases at initial presentation: A systematic review of the literature. Breast Cancer Res Treat 120: 9-16, 2010.

3. Akay CL, Ueno NT, Chisholm GB, Hortobagyi GN, Woodward WA, Alvarez RH, Bedrosian I, Kuerer HM, Hunt KK, Huo L and Babiera GV: Primary tumor resection as a component of multimodality treatment may improve local control and survival in patients with stage IV inflammatory breast cancer. Cancer 120: 1319-1328, 2014

4. Babiera GV, Rao R, Feng L, Meric-Bernstam F, Kuerer HM, Singletary SE, Hunt KK, Ross MI, Gwyn KM, Feig BW, et al: Effect of primary tumor extirpation in breast cancer patients who present with stage IV disease and an intact primary tumor. Ann Surg Oncol 13: 776-782, 2006.

5. Budd GT, Cristofanilli M, Ellis MJ, Stopeck A, Borden E, Miller MC, Matera J, Repollet M, Doyle GV, Terstappen LW and Hayes DF: Circulating tumor cells versus imaging-predicting overall survival in metastatic breast cancer. Clin Cancer Res 12: 6403-6409, 2006.
6. Kim MY, Oskarsson T, Acharyya S, Nguyen DX, Zhang XH, Norton L and Massagué J: Tumor self-seeding by circulating cancer cells. Cell 139: 1315-1326, 2009.

7. Chiotaki R, Polioudaki H and Theodoropoulos PA: Cancer stem cells in solid and liquid tissues of breast cancer patients: Characterization and therapeutic perspectives. Curr Cancer Drug Targets 15: 256-269. 2015.

8. Bafford AC, Burstein HJ, Barkley CR, Smith BL, Lipsitz S, Iglehart JD, Winer EP and Golshan M: Breast surgery in stage IV breast cancer: Impact of staging and patient selection on overall survival. Breast Cancer Res Treat 115: 7-12, 2009.

9. Blanchard DK, Shetty PB, Hilsenbeck SG and Elledge RM: Association of surgery with improved survival in stage IV breast cancer patients. Ann Surg 247: 732-738, 2008.

10. Dominici L, Najita J, Hughes M, Niland J, Marcom P, Wong YN, Carter B, Javid S, Edge S, Burstein H and Golshan M: Surgery of the primary tumor does not improve survival in stage IV breast cancer. Breast Cancer Res Treat 129: 459-465, 2011.

11. Fields RC, Jeffe DB, Trinkaus K, Zhang Q, Arthur C, Aft R, Dietz JR, Eberlein TJ, Gillanders WE and Margenthaler JA: Surgical resection of the primary tumor is associated with increased long-term survival in patients with stage IV breast cancer after controlling for site of metastasis. Ann Surg Oncol 14: 3345-3351, 2007.

12. Gnerlich J, Jeffe DB, Deshpande AD, Beers C, Zander C and Margenthaler JA: Surgical removal of the primary tumor increases overall survival in patients with metastatic breast cancer: Analysis of the 1988-2003 SEER data. Ann Surg Oncol 14: 2187-2194, 2007.

13. Hazard HW, Gorla SR, Scholtens D, Kiel K, Gradishar WJ and Khan SA: Surgical resection of the primary tumor, chest wall control and survival in women with metastatic breast cancer. Cancer 113: 2011-2019, 2008.

14. Khan SA, Stewart AK and Morrow M: Does aggressive local therapy improve survival in metastatic breast cancer? Surgery 132: 620-626; discussion 626-627, 2002.

15. Lang JE, Tereffe W, Mitchell MP, Rao R, Feng L, Meric-Bernstam F, Bedrosian I, Kuerer HM, Hunt KK, Hortobagyi GN and Babiera GV: Primary tumor extirpation in breast cancer patients who present with stage IV disease is associated with improved survival. Ann Surg Oncol 20: 1893-1899, 2013.

16. Neuman HB, Morrogh M, Gonen M, Van Zee KJ, Morrow M and King TA: Stage IV breast cancer in the era of targeted therapy: Does surgery of the primary tumor matter? Cancer 116: 1226-1233, 2010.

17. Pathy NB, Verkooijen HM, Taib NA, Hartman M and Yip CH: Impact of breast surgery on survival in women presenting with metastatic breast cancer. Br J Surg 98: 1566-1572, 2011.

18. Pérez-Fidalgo JA, Pimentel P, Caballero A, Bermejo B, Barrera JA, Burgues O, Martinez-Ruiz F, Chirivella I, Bosch A, Martínez-Agulló A and Lluch A: Removal of primary tumor improves survival in metastatic breast cancer. Does timing of surgery influence outcomes? Breast 20: 548-554, 2011

19. Rapiti E, Verkooijen HM, Vlastos G, Fioretta G, Neyroud-Caspar I, Sappino AP, Chappuis PO and Bouchardy C: Complete excision of primary breast tumor improves survival of patients with metastatic breast cancer at diagnosis. J Clin Oncol 24: 2743-2749, 2006.

20. Rashaan ZM, Bastiaannet E, Portielje JE, van de Water W, van der Velde S, Ernst MF, van de Velde CJ and Liefers GJ: Surgery in metastatic breast cancer: Patients with a favorable profile seem to have the most benefit from surgery. Eur J Surg Oncol 38: 52-56, 2012.

21. Kakarala M and Wicha MS: Implications of the cancer stem-cell hypothesis for breast cancer prevention and therapy. J Clin Oncol 26: 2813-2820, 2008.

22. Danna EA, Sinha P, Gilbert M, Clements VK, Pulaski BA and Ostrand-Rosenberg S: Surgical removal of primary tumor reverses tumor-induced immunosuppression despite the presence of metastatic disease. Cancer Res 64: 2205-2211, 2004.

23. Shien T, Kinoshita T, Shimizu C, Hojo T, Taira N, Doihara H and Akashi-Tanaka S: Primary tumor resection improves the survival of younger patients with metastatic breast cancer. Oncol Rep 21: 827-832, 2009.

24. Ruiterkamp J, Voogd AC, Tjan-Heijnen VC, Bosscha K, van der Linden YM, Rutgers EJ, Boven E, van der Sangen MJ and Ernst MF; Dutch Breast Cancer Trialists' Group (BOOG): SUBMIT: Systemic therapy with or without up front surgery of the primary tumor in breast cancer patients with distant metastases at initial presentation. BMC Surg 12: 5, 2012. 